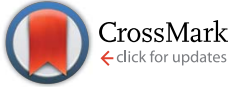

Cite this: RSC Adv., 2017, 7, 13939

Received 6th January 2017

Accepted 7th February 2017

DOI: $10.1039 / \mathrm{c} 7 \mathrm{ra00209b}$

rsc.li/rsc-advances

\section{Real-time and label-free impedimetric analysis of the formation and drug testing of tumor spheroids formed via the liquid overlay technique}

\begin{abstract}
Kin Fong Lei, ${ }^{\text {*abc }}$ Bo-Yuan Lin ${ }^{a}$ and Ngan-Ming Tsang*cd
To achieve high clinical relevance of drug testing, a spheroid culture model is generally used for a standard assay of early tumors. Currently, the quantification of tumor spheroids is generally based on imaging techniques and conventional bioassays. However, additional processing and sophisticated instrumentation are required, which makes high throughput screening infeasible. In this study, tumor spheroids were formed on a thin layer of non-adherent surface (agarose hydrogel), and their responses were monitored by impedance measurements across the interdigitated electrodes fabricated below the hydrogel. The impedance magnitude change was defined as an index to describe the summation effect of the number and size of tumor spheroids. Real-time and label-free impedimetric analysis of the formation and chemosensitivity of tumor spheroids was demonstrated. The technique has the simplicity of device fabrication and experimental processing. Real-time and quantitative assessment could be realized for a routine tool of a spheroid culture.
\end{abstract}

\section{Introduction}

In the discovery and development of anticancer drugs, a success rate as low as $6 \%$ has been reported. ${ }^{1}$ The two-dimensional (2D) cell culture model in which tumor cells grow as a monolayer on glass and plastic substrates is generally adopted and has low predictive value for clinical efficacy. ${ }^{2}$ In recent years, it was reported that using this conventional culture model, it is difficult to mimic the behavior of solid tumors in vivo. ${ }^{3}$ Tumor cells cultured in a three-dimensional (3D) environment are thought to be more physiologically relevant to tumor microenvironment. ${ }^{4}$ Cells proliferate to form multicellular spheroids suspended in a $3 \mathrm{D}$ environment during the culture course. One of the most important differences is that tumor spheroids have higher drug resistance than cells in a monolayer. ${ }^{5,6}$ Consequently, drug testing based on the culture of tumor spheroids has attracted extensive attention and achieved greater predictive capacity. ${ }^{7}$

In spheroid culture models, one of the commonly used methods is to trap and culture tumor cells in the extracellular matrix (hydrogel scaffold). Multicellular tumor spheroids are gradually formed in a 3D environment. ${ }^{89}$ However, this method involves the complex interplay of cells, extracellular matrices, and

${ }^{a}$ Graduate Institute of Medical Mechatronics, Chang Gung University, Taoyuan, Taiwan.E-mail: kflei@mail.cgu.edu.tw; Tel: +886-3-2118800 ext.5345

${ }^{b}$ Department of Mechanical Engineering, Chang Gung University, Taoyuan, Taiwan ${ }^{c}$ Department of Radiation Oncology, Chang Gung Memorial Hospital, Linkou Branch, Taiwan. E-mail: vstsang@cgmh.org.tw; Tel: +886-3-3281200 ext.7140

${ }^{d}$ School of Traditional Chinese Medicine, Chang Gung University, Taoyuan, Taiwan interstitial fluids. Alternatively, to simplify experimental processing, other spheroid culture methods ${ }^{10}$ have been reported including liquid overlay technique, ${ }^{11}$ hanging drop method, ${ }^{12}$ and microfluidic-based methods. ${ }^{13,14}$ Drug testing on tumor spheroids could be performed based on these existing techniques. Generally, analysis of the responses of drug-treated tumor spheroids is based on an imaging approach. The number and size of the tumor spheroids are manually measured under a microscope during the culture course. ${ }^{15,16}$ However, the measurement is subjective and unreliable because of the irregular shape of the spheroids after drug treatment. Moreover, cytotoxicity analysis is conducted by the fluorescence staining method..$^{17,18}$ However, fluorescence dyes often have the problem of non-uniform diffusion into the center of the tumor spheroids. This leads to inaccurate results of drug testing. Alternatively, some research groups utilized conventional bioassays such as MTT assay and flow cytometry to analyze tumor spheroids. ${ }^{19-21}$ These drug efficiency analyses often require additional processing and sophisticated instrumentation, which makes high throughput screening infeasible.

In the past few decades, an impedance measurement technique was applied to quantitatively monitor cellular responses, such as proliferation, migration, and invasion, in a real-time and label-free manner. ${ }^{22-31}$ A comparison of impedimetric analysis of the cellular responses is summarized in Table 1. Most of these studies focused on the cells cultured as a monolayer. ${ }^{23-29}$ Electrodes fabricated on a surface were used and had the advantages of simplicity in terms of device fabrication and experimental processing. Moreover, commercial equipment, such as xCELLigence from ACEA Biosciences and ECIS Z from Applied BioPhysics, were available for real-time monitoring of 
Table 1 Summary of impedimetric analysis of the cellular responses

\begin{tabular}{|c|c|c|c|c|c|}
\hline Cell type & CV-1 and LS174T & BM-1 & BM-1 & Huh-7 & Huh-7 \\
\hline Real-time & Yes & Yes & Yes & Yes & No \\
\hline Culture format & 2D culture & & & $3 \mathrm{D}$ culture & Colonies \\
\hline Application & $\begin{array}{l}\text { Cell growth and } \\
\text { behavior }\end{array}$ & Cell invasion & Cell migration & $\begin{array}{l}\text { Cell growth and } \\
\text { chemosensitivity }\end{array}$ & $\begin{array}{l}\text { Colony formation and } \\
\text { chemosensitivity }\end{array}$ \\
\hline
\end{tabular}

cellular response in a 2D culture model. A number of demonstrations have been reported using this equipment. ${ }^{27-29}$ It is generally agreed that impedimetric analysis provides a convenient and reliable measurement approach for cell-based assays. However, the abovementioned examples consider only a 2D culture model; few studies have attempted to apply impedance measurement technique in a spheroid culture system. Recently, our previous study reported a quantification of the responses of individual cells encapsulated in agarose hydrogel..$^{30}$ A pair of parallel plate electrodes was utilized to measure the total impedance change of the cells/hydrogel construct. However, tumor spheroids could only be cultured from individual cells encapsulated and cultured in soft hydrogels, such as methyl cellulose (MC), because the stiffness of the hydrogel determines the selection and growth of tumorigenic cells. ${ }^{9}$ However, the impedance of MC was too low and a leakage current was induced. This makes the total impedance change in the spheroids/MC construct not sensitive to the responses of tumor spheroids. Hence, impedimetric quantification of tumor spheroids was demonstrated by collecting a small volume $(50 \mu \mathrm{L})$ of spheroids/MC construct and then mixing it with agarose hydrogel for impedance matching. ${ }^{31}$ The mixture was loaded onto a biosensor embedded with a pair of parallel plate electrodes for the end-point measurement. The shortcomings of this technique were as follows: an increase in processing complexity and the sacrifice of cultured spheroids. Real-time and label-free impedimetric analysis of the drug testing of tumor spheroids has not been explored to date.

Tumor spheroids cannot be suspended and cultured in the soft hydrogel; to overcome these challenges, the liquid overlay technique ${ }^{11}$ was adopted to form spheroids on a non-adherent surface in this study. Tumor cells were seeded and cultured on a thin layer of agarose hydrogel (a non-adherent surface). Tumor spheroids were gradually formed on the hydrogel surface and their responses were quantified by impedance measurements across a pair of interdigitated electrodes fabricated under the hydrogel. The proliferation of tumor spheroids could be correlated by the impedance change across the electrodes. In addition, drug testing on tumor spheroids was demonstrated and the dynamic responses under the application of anticancer drugs in different dosages were quantitatively monitored. In conclusion, the technique allows for simple device fabrication and experimental processing. It provides quantitative and objective information to describe the real-time responses of tumor spheroids for higher predictive clinical efficacy.

\section{Materials and methods}

\subsection{Cell culture}

Human hepatoma cells (cell line: Huh7) were kindly provided by Prof. I-Chi Lee at Chang Gung University, Taiwan. The culture medium was Dulbecco's modified eagle medium (DMEM) supplemented with $10 \%$ fetal bovine serum (FBS; Invitrogen, USA) and antibiotic/antimycotic $\left(100 \mathrm{U} \mathrm{mL}^{-1}\right.$ of penicillin G sodium, $100 \mathrm{mg} \mathrm{mL}^{-1}$ of streptomycin, and $0.25 \mathrm{mg} \mathrm{mL}^{-1}$ of amphotericin B; Gibco-BRL Life Technologies, USA). The cells were amplified by a standard cell culture technique and trypsinized using $0.05 \%$ trypsin for $3 \mathrm{~min}$, centrifuged at $1200 \mathrm{rpm}$ for $5 \mathrm{~min}$, and resuspended in the culture medium for further experiments. The number of individual cells was counted using an automated cell counter (Model: Countess II FL; Invitrogen, USA).

\subsection{Tumor spheroids formed via the liquid overlay technique}

The liquid overlay technique was adopted to form tumor spheroids on the hydrogel surface. Agarose hydrogel was used and prepared by mixing $0.5 \%(\mathrm{w} / \mathrm{v})$ agarose powder (Cat No. 50004; Lonza, USA) in the culture medium. After sterilization in an autoclave at $121^{\circ} \mathrm{C}$ under $100 \mathrm{kPa}$ for $20 \mathrm{~min}$, the agarose hydrogel was applied to the culture chamber and it formed a thin non-adherent surface on the bottom of the chamber. The $10^{4}$ cells suspended in the culture medium were then loaded to the culture chamber and cultured in a $5 \% \mathrm{CO}_{2}$ humidified incubator at $37^{\circ} \mathrm{C}$ (Model: 370 ; Thermoscientific, USA). Tumor spheroids were gradually formed on the hydrogel surface during the culture course.

\subsection{Exposure of tumor spheroids to anticancer drugs}

In this study, tumor spheroids were gradually formed on the hydrogel surface in the culture chamber after 2.5 day culture. Starting from the sixtieth hour ( 2.5 days), $20 \mu \mathrm{L}$ of culture medium containing the anticancer drug doxorubicin (Sigma, USA) at the desired dosage was applied to the chamber every $24 \mathrm{~h}$. Doxorubicin induces cell apoptosis and is a commonly used an anticancer drug to treat a wide range of cancers. ${ }^{29}$ The responses of the tumor spheroids were quantitatively investigated based on the drug dosage.

\subsection{Impedimetric analysis of the responses of tumor spheroids}

A biochip composed of 10 culture chambers embedded with interdigitated electrodes was developed for the impedimetric 
analysis of the responses of tumor spheroids. An image of the biochip is shown in Fig. 1(a). The biochip consisted of 2 layers including a polydimethylsiloxane (PDMS; Sylgard ${ }^{\circledR} 184$, Dow Corning, USA) layer constructing 10 culture chambers and a glass substrate with 10 pairs of $\mathrm{Cr} / \mathrm{Au}(300 / 2000 \AA)$ interdigitated electrodes. The size of each chamber was $5 \mathrm{~mm}$ in height and $6.8 \mathrm{~mm}$ in diameter. In each chamber, a pair of interdigitated electrodes was fabricated on the bottom surface and consisted of 23 electrode fingers, as shown in Fig. 1(b). The electrode finger was $150 \mu \mathrm{m}$ in width with a $100 \mu \mathrm{m}$ gap in between, as shown in Fig. 1(c). A pair of electrical contacts was extended to the edge of glass substrate for connection to the impedance analyzer. The PDMS layer was fabricated via soft lithography and the electrodes were fabricated via standard microfabrication techniques. Briefly, a $300 \AA$ chromium layer and a $2000 \AA$ Åld layer were respectively coated on a clean glass substrate via thermal evaporation. Photolithography and $\mathrm{Cr} / \mathrm{Au}$ etching were respectively performed to fabricate the electrodes. Finally, the glass substrates were cleaned by oxygen plasma and bonded with the PDMS layer. Before the experiment, the biochip was washed and sterilized via ultraviolet light overnight. A thin layer of agarose hydrogel was then coated on the bottom of the chamber. Obviously, the thickness of the hydrogel directly influenced the sensitivity of the measurement. The cells suspended in the culture medium were then pipetted in the culture chamber and cultured in an incubator. Tumor spheroids were gradually formed on the hydrogel surface during the culture course. Schematic for the formation of tumor spheroids based on a liquid overlay technique is shown in Fig. 1(b). During the culture course, impedance measurements across the interdigitated electrodes were performed using an impedance analyzer (Model: VersaSTAT 4; Princeton Applied Research, USA). A potential of $0.1 \mathrm{Vrms}$ was applied across the electrodes and the impedance spectrum was obtained from $100 \mathrm{~Hz}$ to 100 $\mathrm{kHz}$. Since the agarose hydrogel was controlled to be thin, the change in the impedance magnitude measured across the electrode was sensitive to the responses of the tumor spheroids. An index of magnitude change was defined to describe the summation effect of the number and size of tumor spheroids.

\subsection{Quantification of tumor spheroids based on a conventional bioassay}

To validate the impedance measurement approach, conventional bioassay was used to quantify the response of the tumor spheroids. The bioassay analyzes the indicative cellular components to
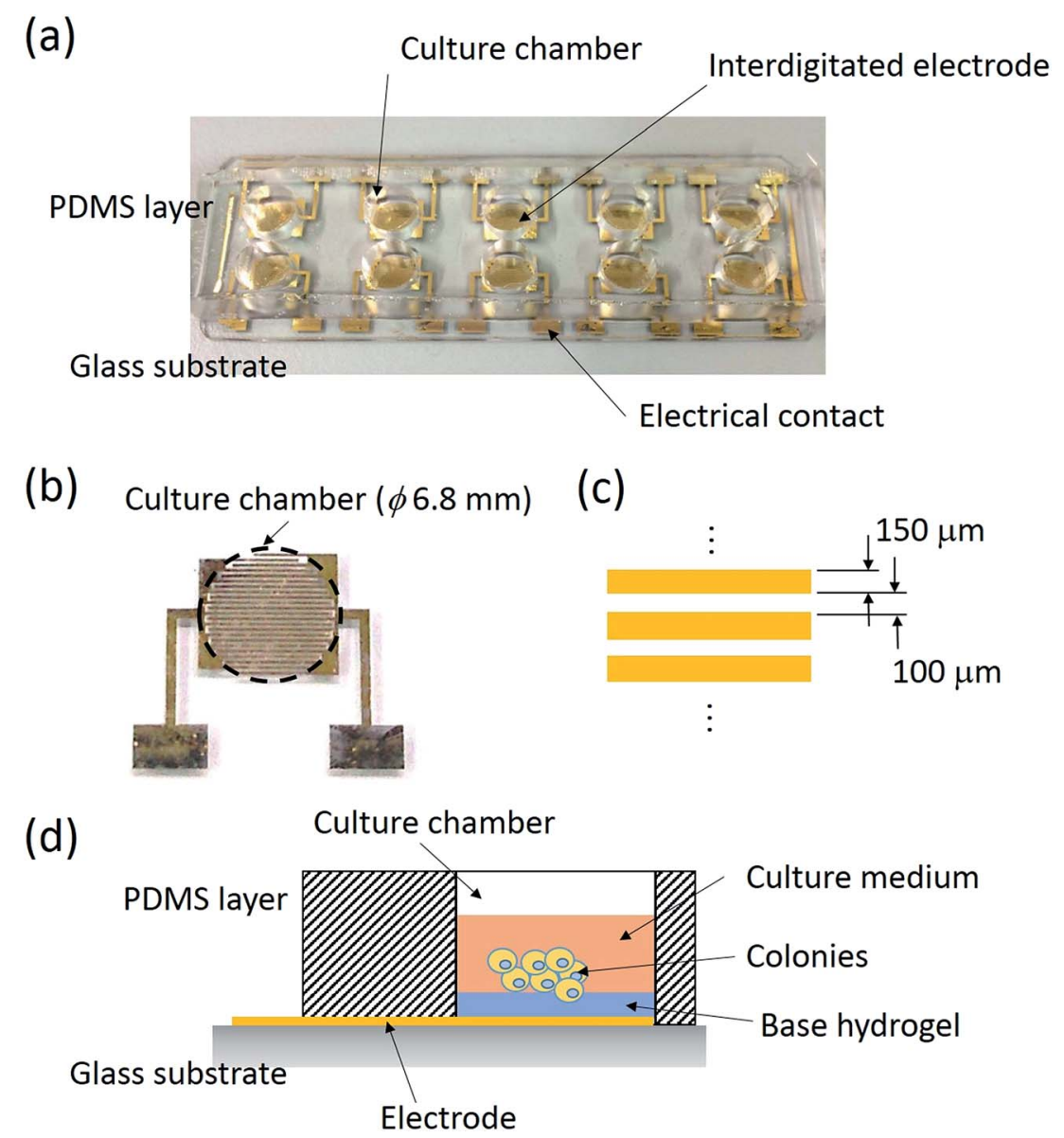

Fig. 1 (a) An image of the biochip. (b) An image of the top-view of a pair of interdigitated electrodes. The dashed circle indicates the location of the culture chamber. (c) Schematic of the dimensions of the electrodes. (d) Schematic for the formation of tumor spheroids based on a liquid overlay technique. Tumor spheroids were formed on the hydrogel surface. 
estimate the cell number. A WST-1 assay (Roche Applied Science, USA) was used in this study. The reagent reacts with the respiratory chain of mitochondria and the color intensity of the reacted product directly correlates with the number of viable cells. To quantify the tumor spheroids, they were collected at days $0,1,3$, and 5 and then broken into individual cells by manual pipetting. Note that 12 identical cultures were prepared for 4 quantification points with 3 repeated measurements because the conventional bioassay requires sacrifice of the culture. Next, the reagent at the volume ratio of $1: 10$ was applied to the cells and incubated for $1.5 \mathrm{~h}$. The color intensity of the reaction product is represented by optical intensity read by a microplate reader (Model: ELx800; BioTek Instruments, Inc., USA) at the absorbance of $440 \mathrm{~nm}$ with the reference wavelength of $660 \mathrm{~nm}$. Therefore, the number of individual viable cells could be correlated with the optical intensity.

\section{Results and discussion}

\subsection{Optimization of the conditions of impedimetric analysis}

In most studies, a spheroid culture was performed via culturing the tumor cells in a 3D environment by total encapsulation in a soft hydrogel. ${ }^{8,9}$ However, because of the issue of impedance matching, the sensitivity of impedimetric analysis is unacceptable for directly quantifying the tumor spheroids suspended in a soft hydrogel. ${ }^{28}$ In this study, the liquid overlay technique was adopted to realize the real-time and label-free analysis of the response of tumor spheroids. To characterize the detection sensitivity, two measurement conditions were investigated including the measurement frequency and thickness of the agarose hydrogel coated on the bottom of the culture chamber. The cells at different cell numbers were applied to the culture chambers coated with $20 \mu \mathrm{L}$ of agarose hydrogel. The thickness of the hydrogel was determined by the pipetted volume. A $20 \mu \mathrm{L}$ of agarose hydrogel was the minimal volume to form the thinnest layer without cell adherence on the chamber. After $6 \mathrm{~h}$ of stabilization, impedance measurements were performed to study the correlation between the impedance magnitude and the cell number at different measurement frequencies of 1,5 , and $10 \mathrm{kHz}$, as shown in Fig. 2(a). A frequency of $1 \mathrm{kHz}$ showed the highest sensitivity (slope of the linear approximation line) and acceptable linearity ( $R$-squared value of the linear approximation line). Frequencies below $1 \mathrm{kHz}$ were unacceptable because of the low signal-to-noise ratio (data not shown). Based on the measurement frequency of $1 \mathrm{kHz}$, the correlations under different thicknesses of agarose hydrogel, such as 20,30, and 40 $\mu \mathrm{L}$, are shown in Fig. 2(b). Generally, the sensitivity increased with the decreasing thickness of agarose hydrogel. Therefore, the optimized measurement conditions of $1 \mathrm{kHz}$ and $20 \mu \mathrm{L}$ of hydrogel were found to well describe the correlation between the impedance magnitude and cell number in this setup.

\subsection{Impedimetric analysis of the formation of tumor spheroids}

Tumor spheroids were gradually formed on the hydrogel surface during the culture course of 5 days. A sequence of (a)

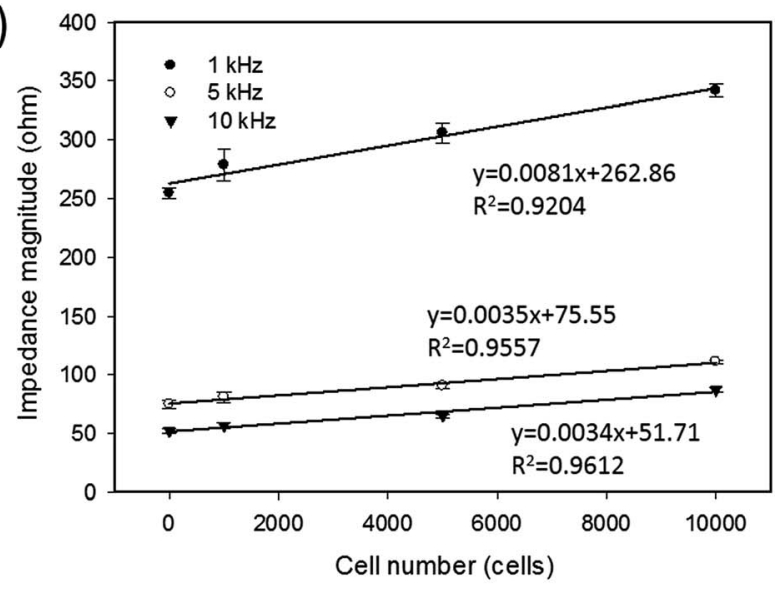

(b)

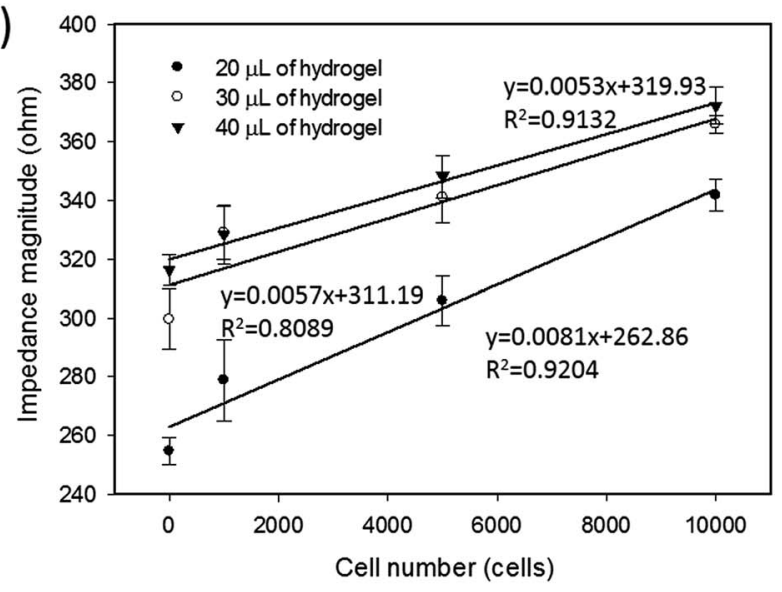

Fig. 2 Correlations between the impedance magnitude and cell number under (a) different measurement frequencies of 1,5 , and 10 $\mathrm{kHz}$ and (b) different thicknesses of hydrogel formed by 20,30, and 40 $\mu \mathrm{L}$ of agarose hydrogel. The error bars represent the standard deviation of 3 repeated measurements.

microscopy images for the formation process is shown in Fig. 3(a). The cells with the diameter of around $10 \mu \mathrm{m}$ proliferated and gradually formed tumor spheroids with a diameter over $200 \mu \mathrm{m}$ during the culture course. As observed from the microscopy images, the sizes of the tumor spheroids were different, especially after day 4 . When tumor spheroids became large, the size variation was significant. Conventional microscopic counting of tumor spheroids was challenged to have objective and accurate results. Hence, impedance measurement was concurrently conducted every $24 \mathrm{~h}$ to quantitatively monitor the formation process. The frequency spectra of the impedance magnitude during the culture course are plotted in Fig. 3(b). It was observed that the equivalent electric circuit of the lumped system was roughly represented by the resistor and capacitor in parallel. As the spheroid proliferated, the impedance magnitudes over the spectrum increased with time. This indicates that most of the contributions were from the increase in the resistance component. This suggests that the tumor spheroids are insulated elements and the formation process can be monitored by impedance measurements. Therefore, magnitude change at the measurement 
(a)

(b)

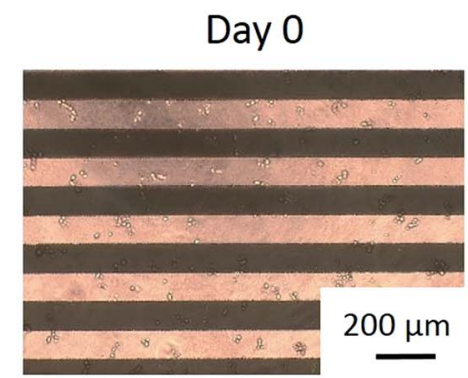

Day 3
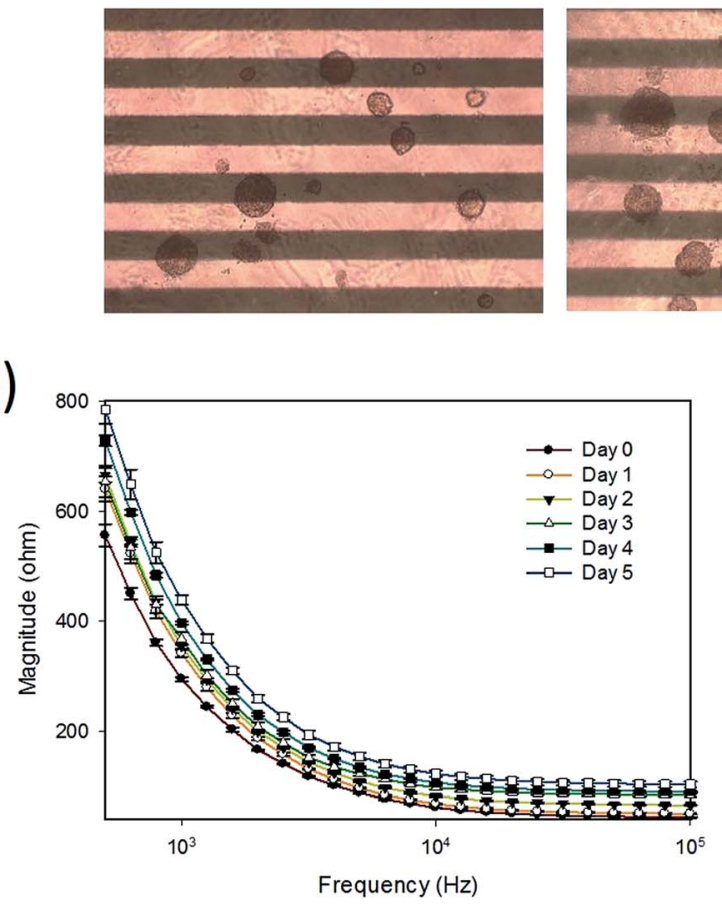

Day 1

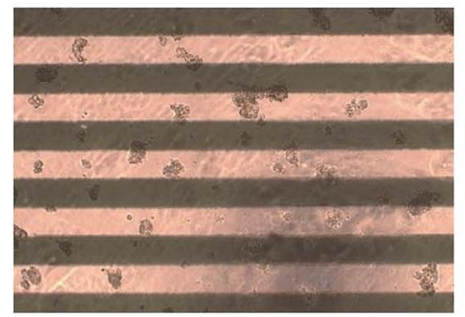

Day 4

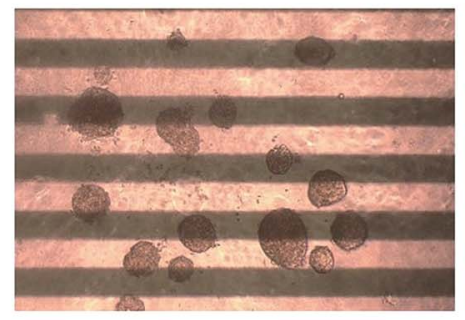

Day 2

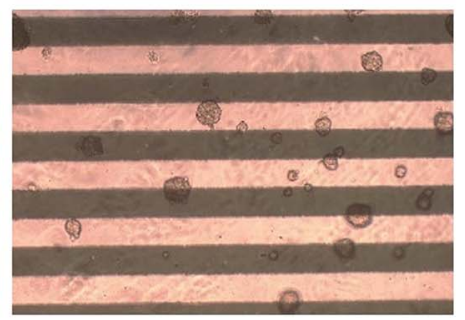

Day 5

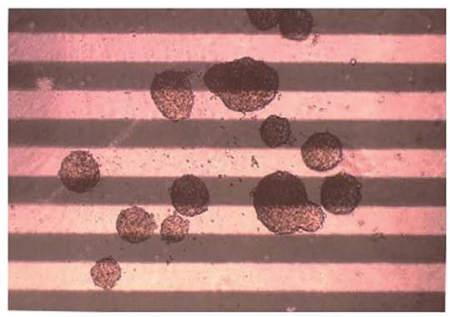

(c)

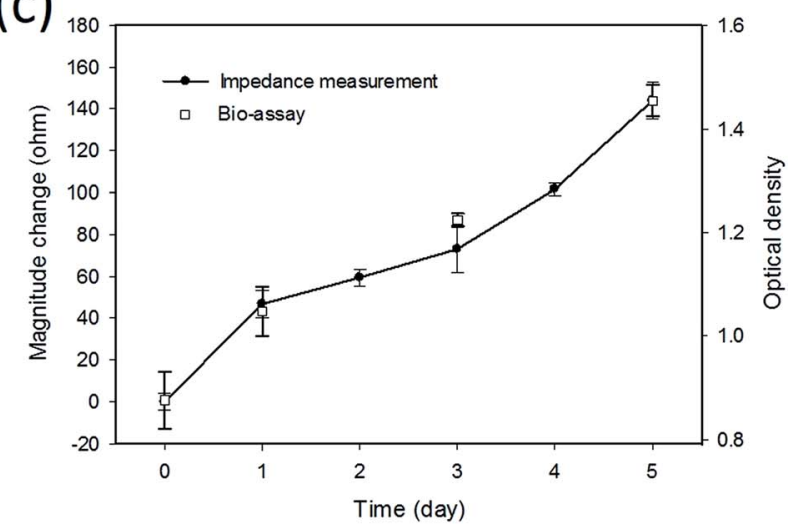

Fig. 3 Impedimetric analysis of the formation process of tumor spheroids. (a) A sequence of microscopic images for the formation process of tumor spheroids during the culture course of 5 days. The parallel bars are the electrodes. (b) Frequency spectra of the impedance magnitude during the culture course. (c) Magnitude change defined as the index for the description of the formation process of tumor spheroids during the culture course. A conventional bioassay was conducted at days $0,1,3$, and 5 to validate the impedimetric results. Error bars represent the standard deviations of 3 repeated measurements.

frequency of $1 \mathrm{kHz}$ was defined as an index to describe the formation process of tumor spheroids, as shown in Fig. 3(c). Compared to the images shown in Fig. 3(a), the image shown in Fig. 3(c) could quantitatively represent the summation effect for the increase in the number and size of tumor spheroids. This is because an increase in cell number induces an increase in the impedance magnitude, as shown in Fig. 2. An increase in the spheroid size also induces an increase in impedance magnitude because the tumor spheroids are insulated elements. In addition, a conventional bioassay was conducted at days $0,1,3$, and 5 to validate the impedimetric results. A high correlation was found between the results generated from impedance measurement and bioassay. This technique was shown to provide quantitative and objective information to describe the formation process of tumor spheroids in a real-time and labelfree manner.

\subsection{Impedimetric analysis of drug testing of tumor spheroids}

In this study, tumor cells were cultured on the hydrogel surface and tumor spheroids were gradually formed in the initial 2.5 days of the culture course. Then, starting from the sixtieth hour (2.5 days), culture media containing doxorubicin at different dosages of 0 (control), 10, 50, and $250 \mu \mathrm{g} \mathrm{mL} \mathrm{mere}^{-1}$ added to the culture chambers every $24 \mathrm{~h}$. Microscopic images of the tumor spheroids were obtained and are shown in Fig. 4. Inhibition of the growth of tumor spheroids was observed in proportion to the drug dosage. During the culture course, impedance measurements were conducted every $12 \mathrm{~h}$ to quantitatively analyze the responses of tumor spheroids in a realtime and label-free manner, as shown in Fig. 5. Generally, the chemosensitivity of tumor spheroids was shown to be dosedependent. The dynamic responses of tumor spheroids were 


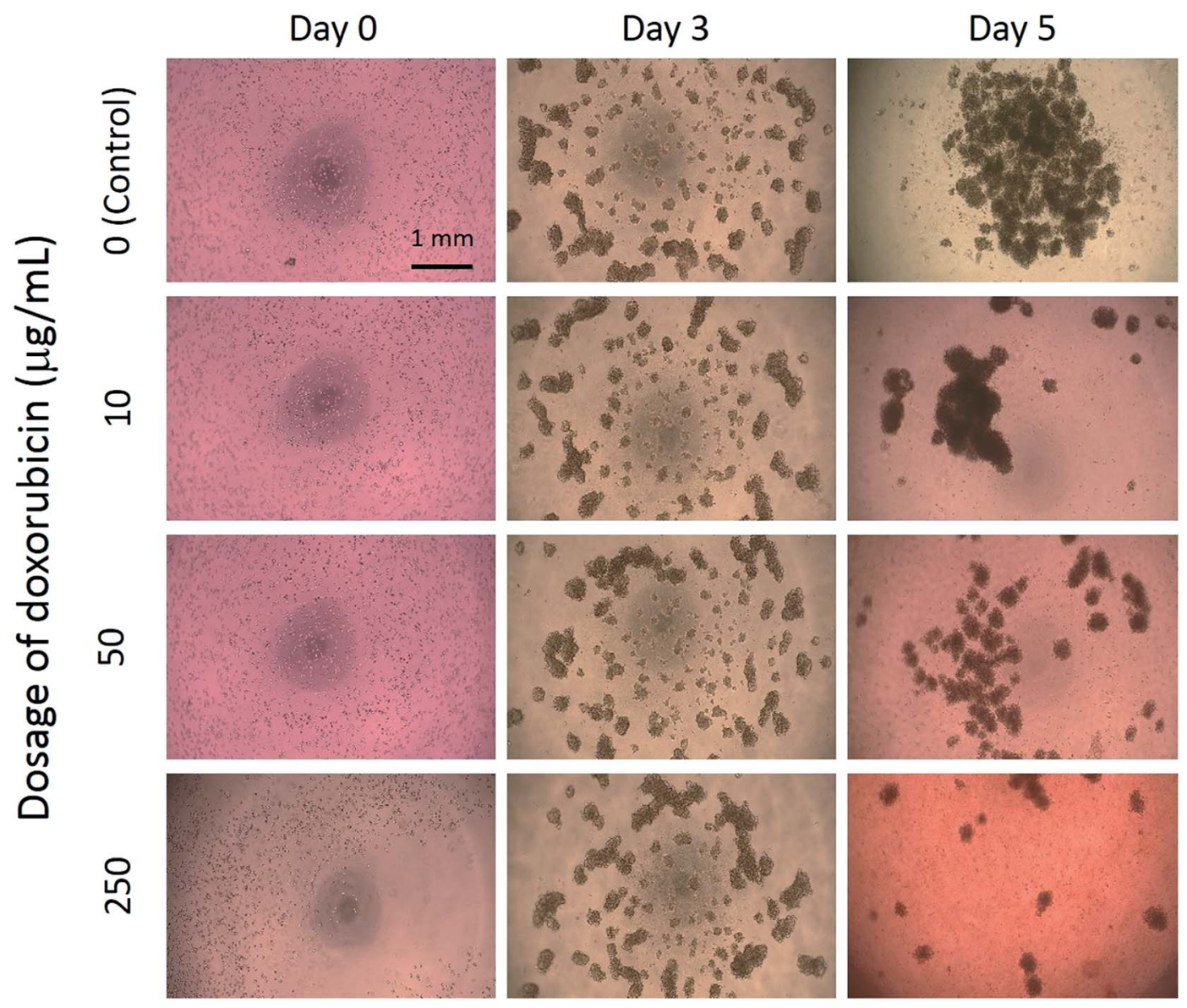

Fig. 4 Microscopic images of tumor spheroids exposed to an anticancer drug at different dosages of 0 (control), 10, 50, and 250 $\mu \mathrm{g} \mathrm{mL}^{-1}$.

obtained and these provided further information of drug efficacy as compared to conventional end-point assays. The results indicated that the growth of tumor spheroids was inhibited by adding the drug at $50 \mu \mathrm{g} \mathrm{mL} \mathrm{m}^{-1}$ and the apoptosis of tumor

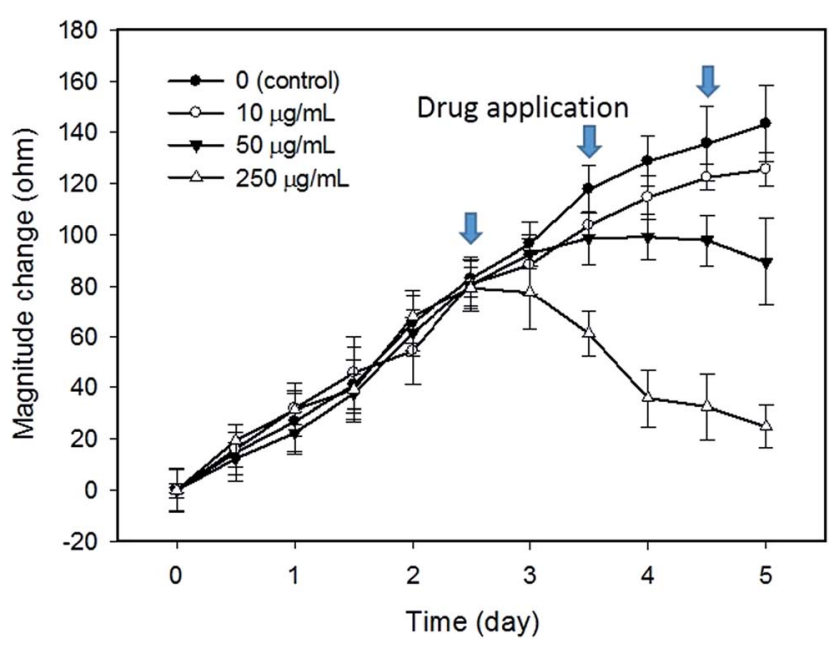

Fig. 5 Impedimetric analysis of tumor spheroids exposed to an anticancer drug at different dosages of 0 (control), 10, 50, and $250 \mu \mathrm{g}$ $\mathrm{mL}^{-1}$. The error bars represent the standard deviations of 3 repeated measurements. spheroids was induced by adding the drug at $250 \mu \mathrm{g} \mathrm{mL} \mathrm{m}^{-1}$. In addition, the relationship between the magnitude changes at day 5 and the drug doses of $0,10,50,250$, and $1000 \mu \mathrm{g} \mathrm{mL} \mathrm{mL}^{-1}$ are plotted in Fig. 6 . Herein, the cell viability of $100 \%$ was obtained on the $5^{\text {th }}$ day of culture without the addition of the drug. In contrast, a cell viability of $0 \%$ was obtained on the $5^{\text {th }}$ day of

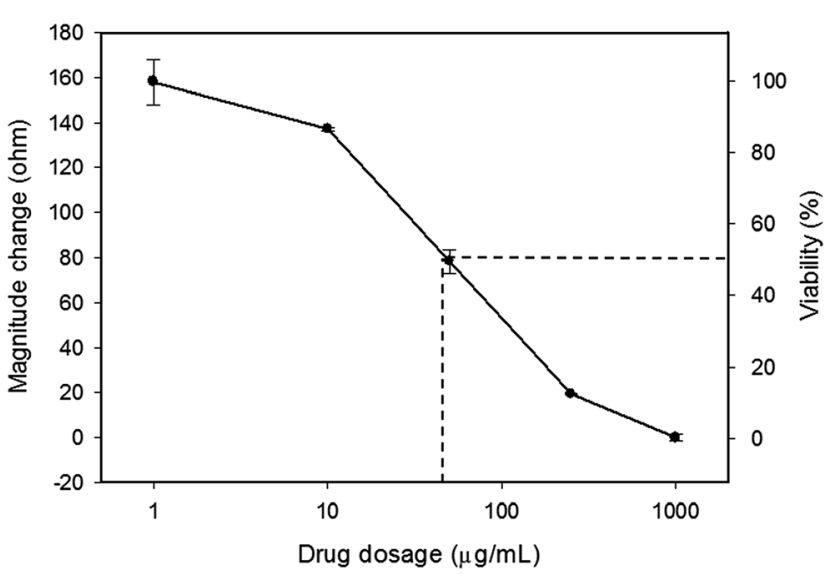

Fig. 6 Dose-response curve of the tumor spheroids after a 5 day culture. The error bars represent the standard deviations of 3 repeated measurements. 
culture containing the drug in $1000 \mu \mathrm{g} \mathrm{mL} \mathrm{m}^{-1}$ (very high dose). Based on the dose-response curve, the $\mathrm{IC}_{50}$ value, which is an important indicator for a chemosensitivity assay, was calculated to be $50 \mu \mathrm{g} \mathrm{mL}{ }^{-1}$. The results confirmed the high drug resistance of the tumor spheroids. This agreed with previous studies reporting that tumor spheroids are more resistant to cytotoxic agents. ${ }^{5,6}$ Compared to that of the cells cultured in a conventional $2 \mathrm{D}$ environment, the $\mathrm{IC}_{50}$ value obtained for the cells cultured in this study was much higher. ${ }^{32}$ Impedimetric analysis of drug testing of tumor spheroids was demonstrated and it showed the feasibility for a quantitative chemosensitivity assay in a real-time and label-free manner.

\section{Conclusions}

In this study, real-time and label-free impedimetric analysis of drug testing of tumor spheroids was successfully demonstrated using interdigitated electrodes. The response of tumor spheroids cultured on a hydrogel surface was well described by the change in impedance magnitude across the electrodes located below the hydrogel. Using a similar hardware setup of commercially available equipment, the impedance measurement could monitor the cellular response from monolayer to 3D spheroid after a thin layer of agarose hydrogel was coated on the electrodes. This approach for analyzing tumor spheroids has the simplicity of processing (without hydrogel encapsulation of cells) and real-time quantification. A higher acceptance of this technique by biologist compared to that of the hydrogel encapsulation of cells is expected. An accurate and informative assessment is important for the drug testing of tumor spheroids and achieving higher success rate for the discovery and development of anticancer drugs.

\section{Acknowledgements}

This study was supported by the Chang Gung Memorial Hospital, Linkou Branch, Taiwan (Project no. CMRPD2F0031 and BMRPC05).

\section{References}

1 M. Hay, D. W. Thomas, J. L. Craighead, C. Economides and J. Rosenthal, Clinical development success rates for investigational drugs, Nat. Biotechnol., 2014, 32, 40-51.

2 F. Pampaloni, E. G. Reynaud and E. H. Stelzer, The third dimension bridges the gap between cell culture and live tissue, Nat. Rev. Mol. Cell Biol., 2007, 8, 839-845.

3 J. Lee, M. J. Cuddihy and N. A. Kotov, Three-dimensional cell culture matrices: state of the art, Tissue Eng., Part B, 2008, 14, 61-86.

4 A. Abbott, Cell culture: biology's new dimension, Nature, 2003, 424, 870-872.

5 V. Koshkin, L. E. Ailes, G. Liu and S. N. Krylov, Metabolic suppression of a drug-resistant subpopulation in cancer spheroid cells, J. Cell. Biochem., 2016, 117, 59-65.

6 P. Longati, X. Jia, J. Eimer, A. Wagman, M. R. Witt, S. Rehnmark, C. Verbeke, R. Toftgård, M. Löhr and
R. L. Heuchel, 3D pancreatic carcinoma spheroids induce a matrix-rich, chemoresistant phenotype offering a better model for drug testing, BMC Cancer, 2013, 13, 95 (13pp).

7 F. Perche and V. P. Torchilin, Cancer cell spheroids as a model to evaluate chemotherapy protocols, Cancer Biol. Ther., 2012, 13, 1205-1213.

8 R. Z. Lin and H. Y. Chang, Recent advances in threedimensional multicellular spheroid culture for biomedical research, Biotechnol. J., 2008, 3, 1172-1184.

9 J. Liu, Y. Tan, H. Zhang, Y. Zhang, P. Xu, J. Chen, Y. C. Poh, K. Tang, N. Wang and B. Huang, Soft fibrin gels promote selection and growth of tumorigenic cells, Nat. Mater., 2012, 11, 734-741.

10 E. Fennema, N. Rivron, J. Rouwkema, C. van Blitterswijk and J. de Boer, Spheroid culture as a tool for creating 3D complex tissues, Trends Biotechnol., 2013, 31, 108-115.

11 E. C. Costa, V. M. Gaspar, P. Coutinho and I. J. Correia, Optimization of liquid overlay technique to formulate heterogenic 3D co-culture models, Biotechnol. Bioeng., 2014, 111, 1672-1685.

12 F. F. Liu, C. Peng, B. I. Escher, E. Fantino, C. Giles, S. Were, L. Duffy and J. C. Ng, Hanging drop: an in vitro air toxic exposure model using human lung cells in 2D and 3D structures, J. Hazard. Mater., 2013, 261, 701-710.

13 K. Ziółkowska, A. Stelmachowska, R. Kwapiszewski, M. Chudy, A. Dybko and Z. Brzózka, Long-term threedimensional cell culture and anticancer drug activity evaluation in a microfluidic chip, Biosens. Bioelectron., 2013, 40, 68-74.

14 Y. C. Chen, X. Lou, Z. Zhang, P. Ingram and E. Yoon, Highthroughput cancer cell sphere formation for characterizing the efficacy of photo dynamic therapy in 3D cell cultures, Sci. Rep., 2015, 5, 12175 (12pp).

15 H. J. Lee, G. Zhuang, Y. Cao, P. Du, H. J. Kim and J. Settleman, Drug resistance via feedback activation of stat3 in oncogene-addicted cancer cells, Cancer Cell, 2014, 26, 207-221.

16 A. Essig, J. Duque-Afonso, S. Schwemmers, H. L. Pahl and M. Lübbert, The AML1/ETO target gene LAT2 interferes with differentiation of normal hematopoietic precursor cells, Leuk. Res., 2014, 38, 340-345.

17 S. Raghavan, M. R. Ward, K. R. Rowley, R. M. Wold, S. Takayama, R. J. Buckanovich and G. Mehta, Formation of stable small cell number three-dimensional ovarian cancer spheroids using hanging drop arrays for preclinical drug sensitivity assays, Gynecol. Oncol., 2015, 138, 181-189.

18 X. Gong, C. Lin, J. Cheng, J. Su, H. Zhao, T. Liu, X. Wen and P. Zhao, Generation of multicellular tumor spheroids with microwell-based agarose scaffolds for drug testing, PLOS One, 2015, 10, e0130348 (18pp).

19 I. C. Lee and J. F. Chang, Label-free selection and enrichment of liver cancer stem cells by surface niches build up with polyelectrolyte multilayer films, Colloids Surf., B, 2015, 125, 120-126.

20 C. C. Yang, L. F. Zhu, X. H. Xu, T. Y. Ning, J. H. Ye and L. K. Liu, Membrane type 1 matrix metalloproteinase induces and epithelial to mesenchymal transition and 
cancer stem cell-like properties in SCC9 cells, BMC Cancer, 2013, 13, 171.

21 B. Patra, C. C. Peng, W. H. Liao, C. H. Lee and Y. C. Tung, Drug testing and flow cytometry analysis on a large number of uniform sized tumor spheroids using a microfluidic device, Sci. Rep., 2016, 6, 21061 (12pp).

22 K. F. Lei, Review on impedance detection of cellular responses in micro/nano environment, Micromachines, 2014, 5, 1-12.

23 I. Giaever and C. R. Keese, Monitoring fibroblast behavior in tissue culture with an applied electric field, Proc. Natl. Acad. Sci. U. S. A., 1984, 81, 3761-3764.

24 R. Ehret, W. Baumann, M. Brischwein, A. Schwinde, K. Stegbauer and K. B. Wolf, Monitoring of cellular behavior by impedance measurements on interdigitated electrode structures, Biosens. Bioelectron., 1997, 12, 29-41.

25 K. F. Lei, H. P. Tseng, C. Y. Lee and N. M. Tsang, Quantitative study of cell invasion process under extracellular stimulation of cytokine in a microfluidic device, Sci. Rep., 2016, 6, 25557 (8pp).

26 L. Liu, X. Xiao, K. F. Lei and C. H. Huang, Quantitative impedimetric monitoring of cell migration under the stimulation of cytokine or anti-cancer drug in a microfluidic chip, Biomicrofluidics, 2015, 9, 034109 (11pp).
27 P. D. Darbre, A. Bakir and E. Iskakova, Effect of aluminium on migratory and invasive properties of MCF-7 human breast cancer cells in culture, J. Inorg. Biochem., 2013, 128, 245-249.

28 C. M. Dowling, C. Herranz Ors and P. A. Kiely, Using realtime impedance-based assays to monitor the effects of fibroblast-derived media on the adhesion, proliferation, migration and invasion of colon cancer cells, Biosci. Rep., 2014, 34, e00126 (17pp).

29 P. Moela, M. M. S. Choene and L. R. Motadi, Silencing RBBP6 (retinoblastoma binding protein 6) sensitizes breast cancer cells MCF7 to staurosporine and camptothecininduced cell death, Immunobiology, 2014, 219, 593-601.

30 K. F. Lei, M. H. Wu, C. W. Hsu and Y. D. Chen, Real-time and non-invasive impedimetric monitoring of cell proliferation and chemosensitivity in a perfusion $3 \mathrm{D}$ cell culture microfluidic chip, Biosens. Bioelectron., 2014, 51, 16-21.

31 K. F. Lei, Z. M. Wu and C. H. Huang, Impedimetric quantification of the formation process and the chemosensitivity of cancer cell colonies suspended in 3D environment, Biosens. Bioelectron., 2015, 74, 878-885.

32 T. K. W. Lee, T. C. M. Lau and I. O. L. Ng, Doxorubicininduced apoptosis and chemosensitivity in hepatoma cell lines, Cancer Chemother. Pharmacol., 2002, 49, 78-86. 